\title{
Eliminating the thermal softening of dynamically loaded specimens in the Kolsky bar system by multi-step loading
}

\author{
Y. Ashuach, C. Avinadav, and Z. Rosenberg \\ Rafael Advanced Defence Systems, PO Box 2250, 31021 Haifa, Israel
}

\begin{abstract}
We performed series of multi-step loading tests in our Kolsky bar system, and demonstrated that the thermal softening in strong aluminum alloys can be eliminated by multi-step loading. We showed that there is a significant difference in their stress-strain curves, compared with the result of a single shot test, due to adiabatic heating. The tests were carried out using our interferometry-based system, where the bar velocities are measured directly rather than the strains. The optical technique has several advantages over traditional strain gauge measurements; it is non-intervening, highly repeatable, and more accurate at low strains, thus allowing good estimation of the dynamic yield point in these experiments.
\end{abstract}

\section{Introduction}

It is well known that materials tested to large strain at high strain rates, by the Kolsky bar system, can experience a significant flow thermal softening. The major part of deformation energy is transferred to heat during these dynamic tests. For materials with relatively low melting point $T_{m}$, this temperature increase can bring the specimen to above 0.4-0.5 $T_{m}$ where the strength of the specimen decreases appreciably. This softening effect can mask and moderate strain hardening, which are observed under quasi-static loading conditions, and can even cause a decrease in the stress-strain curve of the solid. Thus, the dynamic loading by the Kolsky bar system cannot be simply interpreted as a high strain rate test, since it contains, inherently, the thermal softening mechanism. This coupling, between high strain rate and thermal softening, can be prevented by loading the specimen repeatedly, in such a way that the specimen deforms only slightly during each loading. In this study we performed a series of tests with multiply loaded specimens, made of different aluminum alloys, and compared their stress-strain response to that achieved by a single high strain loading. As expected, we found that due to lack of thermal softening, the stress-strain curve from the multi-step test is higher than that from the one-shot test.

The tests were carried out using our new, highly accurate technique, for measuring the dynamic strains in the Kolsky bar, as described in [1]. Our method is based on interferometeric measurement of the bar's velocity $v(t)$ which, in the elastic region, is directly proportional to the strain $\varepsilon(t)$ through:

$$
\varepsilon(t)=\frac{1}{c_{0}} v(t),
$$

where $c_{0}$ is the longitudinal speed of sound in the bar. Engin eering strain, stress and strain rate are calculated from the well-known equations derived by Kolsky [2], replacing measured strains with velocities:

$$
\sigma(t)=\frac{E_{0}}{c_{0}} \frac{A_{0}}{A_{s}} v_{T}(t)
$$

$$
\begin{gathered}
\varepsilon(t)=-\frac{2}{L} \int_{0}^{t} v_{R}(t) d t \\
\dot{\varepsilon}(t)=-\frac{2}{L} v_{R}
\end{gathered}
$$

where $E_{0}$ is the elastic modulus of the bars, $A_{0}$ and $A_{S}$ are the cross-sectional area of the bars and specimen, respectively, and $v_{T}$ and $v_{R}$ are the transmitted and reflected velocities as measured by the interferometer.

The bars are probed by laser beams, making the measurement non-intervening and insensitive to the impacts in the system. Moreover, the velocity data is encoded in the frequency domain of the measured signals, making them insensitive to electrical noise. Thus, the optical measurement is significantly more reliable than the conventional strain gauge technique.

In the next section we describe the optical technique for the Kolsky bar apparatus, including a description of the interferometer system, its integration in the Kolsky bar apparatus, and the analysis of the recorded signals. In section 3 we show some experimental results from the tests with aluminum alloys demonstrating the diminution of thermal softening due to the multiple loadings.

\section{The optical Kolsky bar apparatus}

\subsection{Description of the apparatus}

Figure 1 shows our interferometry-based Kolsky bar apparatus. In our setup, the interferometers are used as nonintervening replacements for the strain gauges. They measure the velocities at the sides of the bars, approximately at the same locations where the strain gauges are usually mounted. Two fiber focusers are set to illuminate the incident and transmitter bars at a $30^{\circ}$ angle relative to the bars. For comparison purposes, two strain gauges (EA-06250BG-120 manufactured by Micro-Measurements) are also glued to the incident and transmitter bars. A third optical measurement is optionally performed on the specimen itself, measuring its radial expansion rate at an angle of 


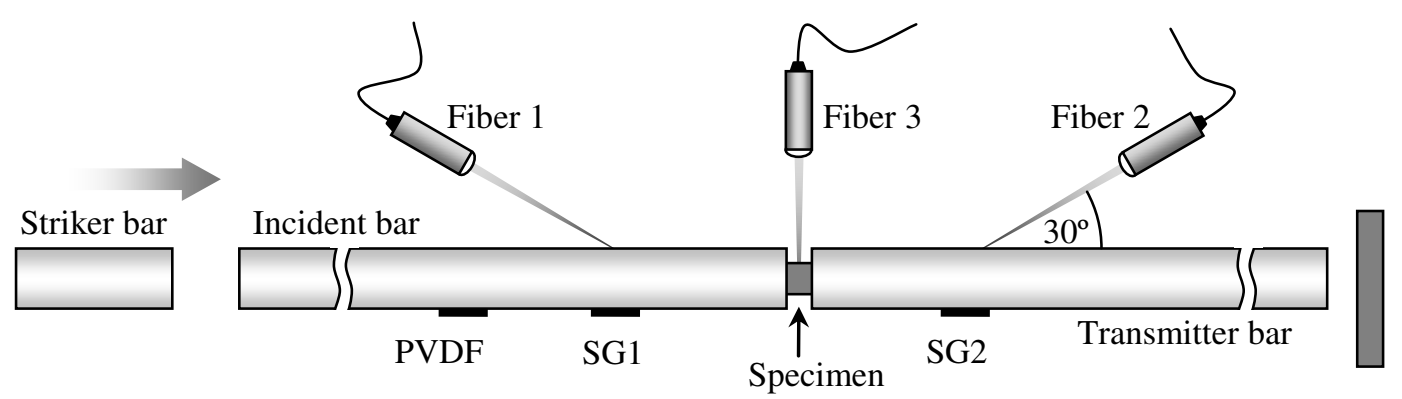

Fig. 1. Our optics-based Kolsky bar apparatus, with two or three fiber focusers, a PVDF foil transducer for triggering, and optional strain gauges (SG).

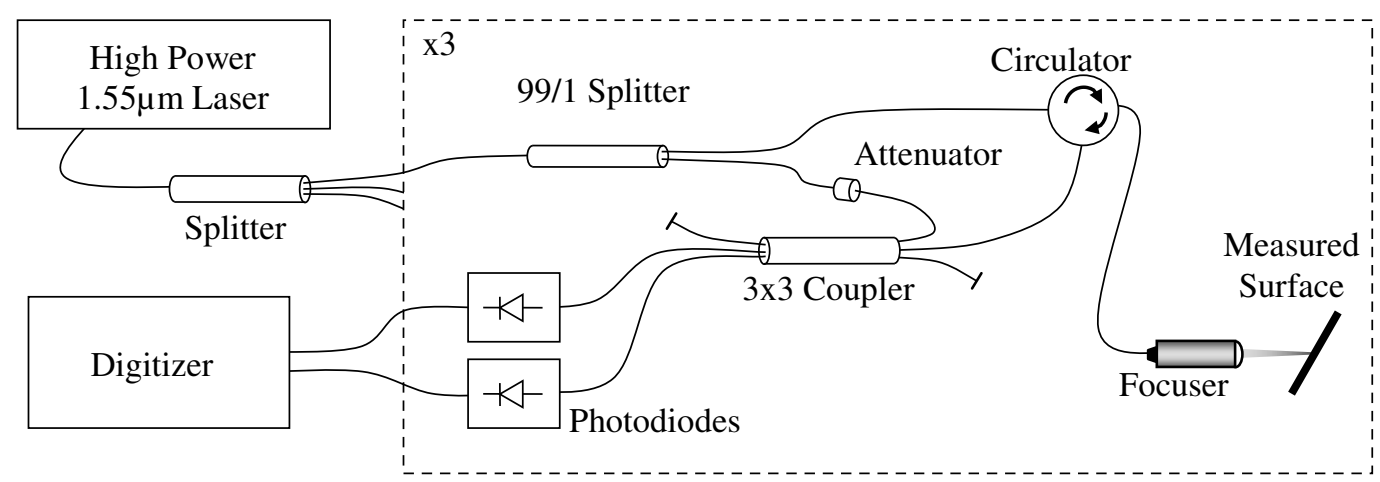

Fig. 2. The optical setup of the interferometeric measurement. See text for detailed explanation.

$90^{\circ}$. Finally, a PVDF electrode is attached to the incident bar and provides a triggering signal for data acquisition.

We use standard pigtailed fiber focusers manufactured by $\mathrm{Oz}$ Optics, designed to a $50 \mathrm{~mm}$ working distance and a $75 \mu \mathrm{m}$ spot size. In order to enhance backscattering from the semi-specular surface of the bars, we apply a white paint on the bars at the measurement locations. This procedure was proven to create diffusive reflection which increases the optical collection efficiency.

The optical measurement in our system is possible due to the fact that photon Doppler velocimetry does not necessarily measure the phase changes resulting from the movement of the reflecting surface through the beam. In our case, these phase changes would be approximately zero. Rather, it has been shown that when measuring diffuse surfaces, the recorded frequency is due to the Dopplershifted velocity component along the beam direction $[3,4]$. As a result, our technique measures the velocity of the compressing bars simply by reflecting light off them at a small angle.

The relation between the actual velocity $v_{\text {real }}$ of the bars and the measured velocity $v_{\text {meas }}$ is simply

$$
v_{\text {real }}=\frac{v_{\text {meas }}}{\cos \theta}
$$

where $\theta$ is the angle between the laser beam and the bars. As a result, the most prominent source of systematic error in determining the velocities of the bars is the accuracy in determining $\theta$. Working with an angle of $30^{\circ}$, we have $\delta v / v \approx 0.7 \delta \theta$, therefore special care must be taken in order to ensure that the total pointing error of the fiber focusers and their alignment assembly is sufficiently small. The focusers we use have an inherent pointing error of about $10 \mathrm{mrad}$, and our holder assembly has an alignment error which is much smaller. Therefore, we estimate the absolute accuracy of our velocity measurement at about $0.7 \%$, which can be improved by using focusers with better pointing accuracy or by mounting them at a smaller angle.

Note that as the bar is compressed longitudinally it also expands radially, creating a radial velocity component which contributes to the recorded signal. This velocity is proportional to the derivative of the longitudinal velocity, which oscillates around zero during the measurement, with typical amplitudes of the order of $0.1 \mathrm{~m} / \mathrm{s}$. Thus, the measured longitudinal velocity is practically not affected by the bar expansion.

\subsection{Description of the optical measurement system}

We implemented an all-fiber velocity interferometer, shown in figure 2, which is based on the design of the recently developed Photonic Doppler Velocimetery (PDV) system by Strand [5]. Unlike his system, our implementation of the PDV is designed for measuring very low velocities, of the order of several m/s, for which lower-bandwidth photodetectors and digitizers are sufficient. Additionally, Kolsky bar tests are characterized by fast varying low velocities, which are difficult to analyze with single phase measurements. Thus, our system incorporates a $3 \times 3$ single-mode fiber coupler that readily creates three interferometric signals which are $120^{\circ}$ out of phase with each other [6,7]. A commercial high-power $1.55 \mu \mathrm{m}$ fiber laser is used, whose output is split into two or three independent interferometers, each using about $1 \mathrm{~W}$ of laser power.

This setup, which is analogous to a traditional MachZehnder interferometer, produces oscillating signals with 

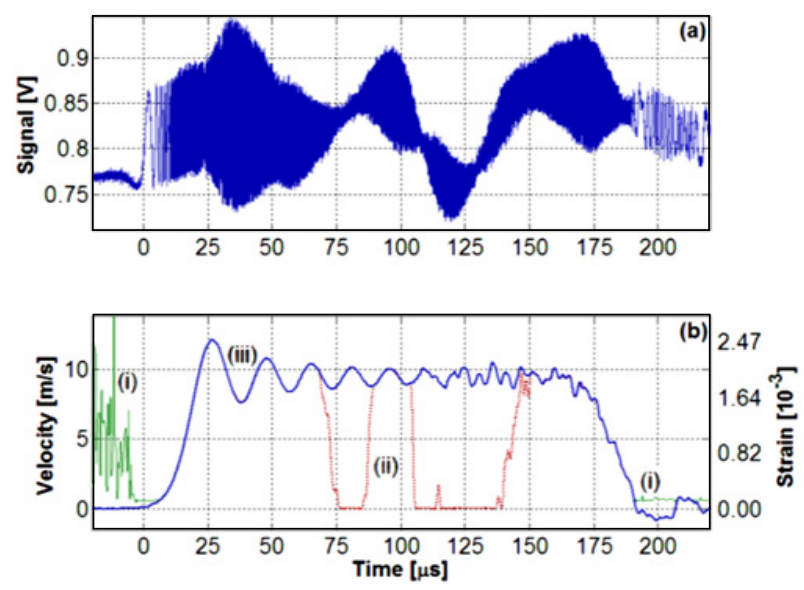

Fig. 3. a) Raw interferometer data, showing variations in signal amplitude and baseline. (b) calculated velocity using the Fourier method (i), the phase analysis method (ii) and their combined result (iii).

an instantaneous frequency equal to the optical Doppler shift of the reflected target light,

$$
f=2 v / \lambda,
$$

where $\lambda=1.55 \mu \mathrm{m}$ is the laser wavelength. For typical Kolsky bar experiments the striker velocity is of the order of $5-20 \mathrm{~m} / \mathrm{s}$, therefore the required bandwidth for the photodiodes and digitizers is $\sim 10-30 \mathrm{MHz}$.

The light intensity which reaches the photodiodes is given by

$$
\begin{aligned}
& I_{1}(t)=\frac{1}{3}\left[I_{\text {ref }}+I_{\text {target }}+2 \sqrt{I_{\text {ref }} I_{\text {target }}} \cos (2 \pi f t)\right] \\
& I_{2}(t)=\frac{1}{3}\left[I_{\text {ref }}+I_{\text {target }}+2 \sqrt{I_{\text {ref }} I_{\text {target }}} \cos \left(2 \pi f t+\frac{2}{3} \pi\right]\right.
\end{aligned}
$$

where $I_{\text {ref }}$ and $I_{\text {target }}$ are the light intensities of the reference and target beams, respectively. The intensity factor $(1 / 3)$ and the phase differences $(2 \pi / 3)$ are due to the $3 \times 3$ fiber coupler. Usually we set the reference beam intensity to about $0.5-1 \mathrm{~mW}$, and the collected target beam intensity is typically $10-20 \mu \mathrm{W}$. The photodiodes have a transimpedance amplification of $5 \mathrm{k} \Omega$ and a sensitivity of nearly $1 \mathrm{~A} / \mathrm{W}$, which results in signals with a typical DC offset (due to $I_{\text {ref }}$ ) of $1-2 \mathrm{~V}$ and an oscillation amplitude of $0.25-0.5 \mathrm{~V}$.

\subsection{Method of analysis}

There are two methods for the analysis of heterodyne interferometric data, as described below. Each of these methods has its benefits and drawbacks, and both of them are applied for different parts of the recorded signal, in order to obtain a single time-velocity record in each test. The complexity of the analysis, and the need for two methods, can be understood through the characteristics of the measured interference signal, shown in figure 3(a). Its frequency changes continuously from zero to $\sim 15 \mathrm{MHz}$; the amplitude varies in time due to changes in the amount of light that is collected by the fiber focusers; and non-ideal fiber components (primarily the circulator) allow parasitic non-Doppler shifted light to interfere with the reference signal, inducing slow baseline changes in the signal.

The first method is based on a short-time Fouriertransform (STFT) [8]. This is the regular procedure for the analysis of PDV data [9], based on estimating the instantaneous signal frequency from the time-frequency spectrogram. We use $2.56 \mu$ s-long Hamming windows and $1.92 \mu$ s overlap between consecutive windows $(0.64 \mu \mathrm{s}$ step size). Sub-bin resolution is obtained by fitting the spectral peak to a Gaussian-like function.

The second method is based on phase-sensitive analysis of the recorded data. This analysis, unlike the Fourier method, requires two out-of-phase measurements of the interference signal. If the relative phase between the two measurements is known, the absolute phase of the interference signal can be extracted by quadrature phase analysis [10]. The phase is unwrapped and the displacement of the reflecting surface is calculated through

$$
\Delta x(t)=\frac{\lambda}{2} \frac{\Delta \phi(t)}{2 \pi}
$$

This displacement signal is then numerically differentiated to obtain the velocity record. The phase analysis is sensitive to baseline changes in the signals, and therefore these are filtered by subtracting an averaged version of the signal from itself. Typically, the phase-sensitive analysis is used with a $40 \mu \mathrm{s}$ averaging window at the baseline removal stage. The displacement data is also averaged before and after the numerical derivative is calculated, with a $1 \mu$ s averaging period at both stages.

The analysis through the Fourier method is more robust than the phase-sensitive analysis; it is able to extract reliable velocity information even at relatively low signal-to-noise values and is insensitive to amplitude and baseline changes. However, the Fourier analysis fails to analyze fast-varying low-velocities $(<1 \mathrm{~m} / \mathrm{s})$, i.e. when the instantaneous frequency of the signal changes at a rate comparable to the frequency itself. This situation often occurs in Kolsky bar tests, due to the slow rise-time of the velocity signals on both bars. On the other hand, the phasesensitive analysis gives reliable results at the low-velocity regime, which is typically characterized by high signal-tonoise ratio (SNR) and negligible amplitude and baseline variations. In regions where both velocity and SNR are high, the two methods agree to within $0.05 \mathrm{~m} / \mathrm{s}$.

Following these considerations, the analysis of the recorded signals is done with both methods, see figure 3(b). The velocity is first calculated using both methods in an automatic routine that uses the typical parameters listed above (windows size, overlap, etc.), which were found suitable for all of our experimental data. A single velocity trace is then constructed from the two analyzed results, by manually choosing the transition points between low and high velocity regions. Finally, the recorded velocities are used to calculate stress and strain histories through equations (2)-(4). 


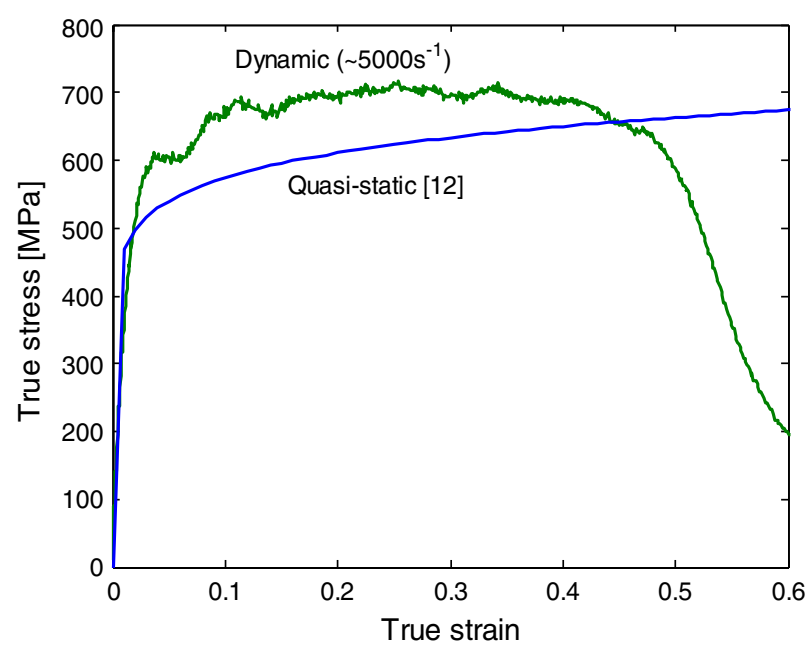

Fig. 4. Stress-strain curves of aluminum 7075-T6 specimens under quasi-static and dynamic loading.

\section{Multi-step loading tests}

The temperature increase due to plastic deformation is estimated by the well-known Taylor-Quinney relation:

$$
d T=\frac{\kappa \cdot \sigma \cdot d \varepsilon}{\rho \cdot c_{v}}
$$

where $\sigma$ and $\varepsilon$ are the stress and strain, $\rho$ is the material density, $c_{v}$ is its specific heat capacity, and the TaylorQuinney coefficient $\kappa$ is the fraction of energy transferred to heat. Recent estimates for the actual value of this constant are: $\kappa \cong 0.95-1.0$, as discussed in [10] and [11], for example. It is clear from eq. (9) that a significant temperature rise can be expected for low density materials, having high strength, that are loaded to high strains. High strength aluminum alloys, such as the 7075-T6 alloy, are possible candidates for such a significant thermal softening effect.

In order to demonstrate this issue we consider the two stress-strain curves for 7075-T6 aluminum as shown in figure 4, which were obtained by quasi-static loading [12] and the dynamic loading in our Kolsky bar system. The quasi-static curve shows a gradual strain hardening effect up to the highest strains. On the other hand, the dynamic curve reaches a plateau at a strain if about 0.3 and it decreases at higher strains. This is probably due to the combined effect of the strain hardening and the thermal softening which take place in the dynamic loading of the specimen. At a strain of about $0.3-0.4$ the flow stress reaches its maximal value as a result of the two conflicting trends.

For quantitative estimation of the thermal softening effect in these experiments, consider the 6061-T6 aluminum alloy, having a flow stress of about $400-420 \mathrm{MPa}$ up to strain of 0.8 . Using an average value of $410 \mathrm{MPa}$, eq. (9) results in a temperature rise of about $130^{\circ} \mathrm{C}$. This means that at the end of the Kolsky bar test the specimen reaches $423 \mathrm{~K}$, which is roughly $45 \%$ from the melting temperature $\left(T_{m}=923 \mathrm{~K}\right)$.

In order to verify these estimates, consider the results of Rosenberg et al. [13] for the flow stress of heated

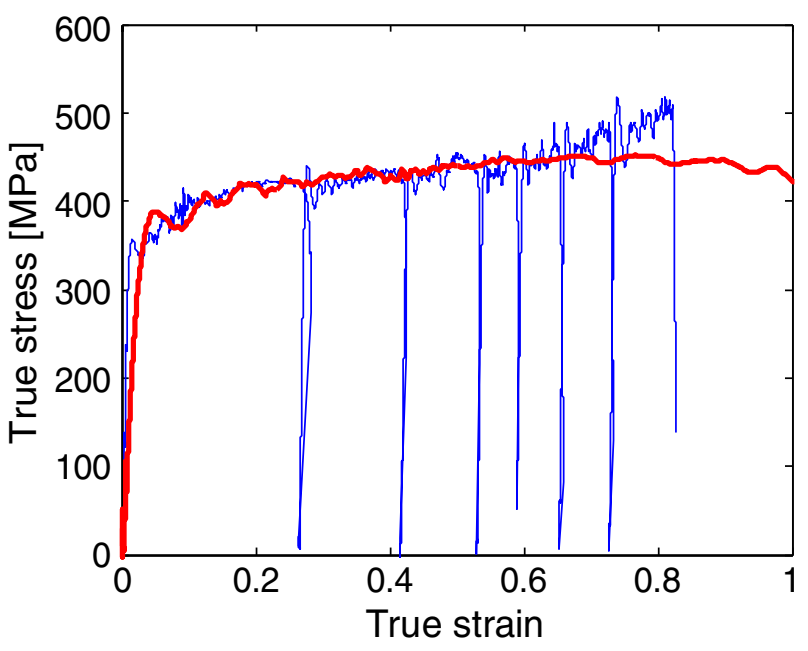

Fig. 5. Stress-strain curves from tests with aluminum 6061-T6 specimens under multi-step and one-shot loading.

6061-T6 specimens in the Kolsky bar system. It is clear from their results that a temperature increase of about $120^{\circ} \mathrm{C}$ should not result in a drastic change in the flow stress of this alloy. However, the decrease in flow stress is not linear, and it is more significant above $200^{\circ} \mathrm{C}$, which is roughly $0.5 T_{m}$.

Figure 5 shows the result from the one-shot test vs. the results from the multi-step tests, for aluminum 6061-T6 sample. The initial dimensions of the specimens were the same: diameter of $10 \mathrm{~mm}$ and thickness of $5 \mathrm{~mm}$. The two curves practically coincide up to a strain of $\sim 0.6$. At higher strains, the one-shot curve flattens to a plateau, while the multi-step curve continues rising. This small difference may be the result of the temperature increase of about $130^{\circ} \mathrm{C}$ in the specimen which experienced a single loading, as calculated above.

Clearly, the temperature rise during each step in the multiply-loaded specimen is negligible, since the strains in these steps were of the order of $0.1-0.25$. The fact that the two curves coincide at the low strain region means that there is no strain rate hardening at the strain rate region from $500 \mathrm{~s}^{-1}$ (of the multi-step tests) to $7000 \mathrm{~s}^{-1}$ (of the single shot).

Another issue to note about the stress-strain curve in figure 5 is that our optical measurement succeeded better than the strain gauges in highlighting the yield stress of this aluminum alloy. The stress-strain curve for this material shows a clear change in its slope at about $350 \mathrm{MPa}$, which is very similar to the yield stress of $345 \mathrm{MPa}$ measured by Luk et al. [14] at a much lower strain rate of $10^{-1} \mathrm{~s}^{-1}$. This agreement enhances the claim that the 6061-T6 alloy is insensitive to strain rate at the range of strain rates of $10^{-1}-10^{4}$.

The temperature rise due to the adiabatic nature of the deformation should be larger for aluminum alloys which have a higher strength. For example, the 2024-T351 alloy, with a flow stress in the range of 500-600 MPa, as shown in figure 6, should be heated to around $480 \mathrm{~K}$ at strains of about 0.5 . Assuming that the strength reduction of these aluminum alloys, due to thermal softening, is similar, we can use the data from [13] for the 2024 alloy as well. 


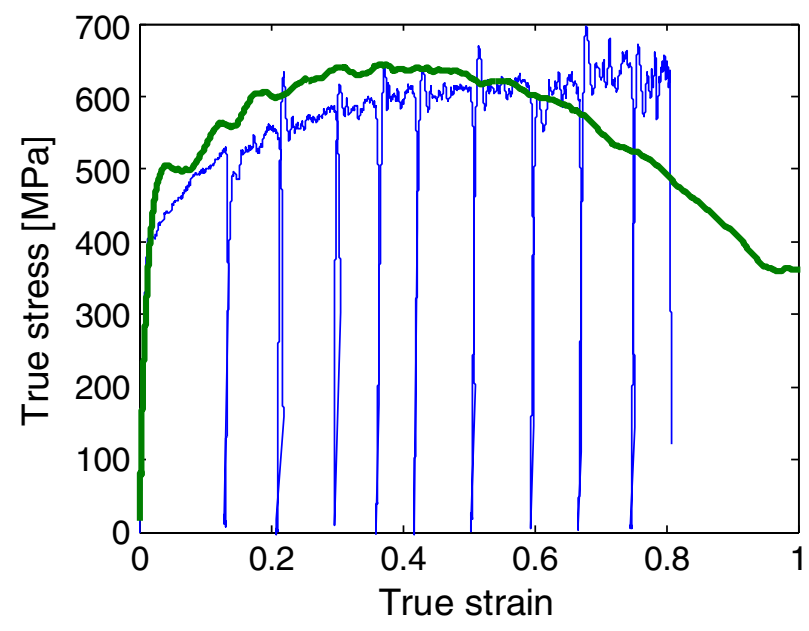

Fig. 6. Stress-strain curves from tests with aluminum 2024T351 specimens under multi-step and one-shot loading.

Thus, a temperature increase of nearly $200^{\circ} \mathrm{C}$ should yield a reduction of the flow stress by about $100 \mathrm{MPa}$, which amounts to $20 \%$ of the flow stress.

Results from tests with this alloy are shown in figure 6 . Clearly, the two loading modes resulted in very different stress-strain curves. The multiply loaded specimen shows a constant increase of its flow stress, due to strain hardening. On the other hand, the specimen loaded by a single step shows the contradicting influences of strain hardening and thermal softening, which lead to the decrease in flow stress at a strain of about 0.5. We expect a same trend for specimens made of the 7075-T6 alloy.

The vertical shift in the values of the flow stresses, at the low strain values, can be explained by strain rate hardening for the 2024 alloy. The striker velocity in the single step experiment was much higher than the velocities in any of the steps of the multi-step test. Thus, while the single step test was performed at a strain rate of about $7000 \mathrm{~s}^{-1}$, each test in the multi-step series was done at a strain rate in the range of $500-1000 \mathrm{~s}^{-} 1$. The result of this difference is the vertical shift between the two curves at the low-strain region.

\section{Conclusions}

We have demonstrated a new experimental approach for the Kolsky bar system, which is based on optical velocity measurements. Two analysis methods of the interferometric measurements were presented, which are combined to determine the accurate bar velocity record even at very low strains. The optical technique has several advantages over traditional strain gauge measurements; it is nonintervening, highly repeatable, and more accurate at low strains.

From series of multiple loading tests in our Kolsky bar system, we have demonstrated that one can delineate the inherent temperature rise in dynamic experiments. We chose two aluminum alloys with different strengths and showed that there is a significant difference in their stressstrain curves due to adiabatic heating. Using this method of multiple loading gives the inherent sensitivity of the specimen to the high loading rates and large strains.

The optical interferometric technique which we used in this study has been shown to be a very convenient and accurate one. In particular, the small size of the laser spot $(75 \mu \mathrm{m})$ enables one to distinguish between the elastic and plastic parts of the dynamic stress-strain curve, as determined by the Kolsky bar system. This is a very important property which can be extracted from this optical system, which was not possible with the strain gauge records.

\section{References}

1. C. Avinadav, Y. Ashuach, R. Kreif, Rev. Sci. Instrum. 82, 073908 (2011)

2. H. Kolsky, Proc. Phys. Soc. London B, 62(11), 676 (1949)

3. M. E. Briggs, L. M. Hull, and M. A. Shinas, Fundamental experiments in velocimetry, AIP Conf. Proc. 1195, 577 (2009)

4. D. H. Dolan, AIP Conf. Proc. 1195, 589 (2009)

5. O. T. Strand, Rev. Sci. Instrum. 77, 083108 (2006)

6. R. G. Priest, IEEE T. Microw. Theory, MTT-30[10], 1589 (1982)

7. M. A. Choma, C. Yang, and J. A. Izatt, Opt. Lett. 28, $22(2003)$

8. D. Gabor, J. IEE 93, 429 (1946)

9. D. H. Dolan and S. C. Jones, Rev. Sci. Instrum. 78, 076102 (2007)

10. E. El Magd, M. Abouridouane, J. Phys. IV France 110, 15 (2003)

11. P. R. Dixon, D. J. Parry, J. de Phys. IV C3-85 (1991)

12. M. Forrestal, V. K. Luk, Z. Rosenberg, N. S. Brar, Int. J. Solids Structures 29, 1729 (1992)

13. Z. Rosenberg, D. Dawicke, E. Strader, S. J. Bless, Exp. Mech. 26, 275 (1986)

14. V. K. Luk, A. J. Piekutowski, Int. J. Impact Engng. 11, 323 (1991) 